\title{
From Shell Logs to Shell Scripts
}

\author{
Nico Jacobs and Hendrik Blockeel \\ Katholieke Universiteit Leuven, \\ Department of Computer Science, \\ Celestijnenlaan 200A, B-3001 Leuven \\ tel +3216327550 \\ fax +3216327996 \\ $\{$ Nico.Jacobs, Hendrik.Blockeel\}@cs.kuleuven.ac.be
}

\begin{abstract}
Analysing the use of a Unix command shell is one of the classic applications in the domain of adaptive user interfaces and user modelling. Instead of trying to predict the next command from a history of commands, we automatically produce scripts that automate frequent tasks. For this we use an ILP association rule learner. We show how to speedup the learning task by dividing it into smaller tasks, and the need for a preprocessing phase to detect frequent subsequences in the data. We illustrate this with experiments with real world data.
\end{abstract}

Keywords: sequences, adaptive user interface

\section{By your command}

For many years now, the Unix command shell ${ }^{1}$ is used by experienced and inexperienced users to interact with their system. Although this shell exists for many years, it can still be considered a flexible and customisable user interface: aliases allow you to give easier names for certain commands, with scripts you can build new commands by combining existing commands, et cetera. Many users do not fully utilise the power of the shell, either because they do not know all the tools the shell can provide or they do not want to go through the effort of using the tools.

Since the shell is such a complex and powerful user interface many people have investigated how users use this shell. Greenberg [6] collected logs from 168 different users, classified in four categories: computer scientists, experienced programmers, novice programmers and users performing no programming tasks (such as people from the administration et cetera). He used the standard statistical techniques to analyse e.g. which history mechanism is most useful.

Later on, people start using machine learning techniques to automatically analyse logs of shell use. One way to help people using the shell is by predicting the next command they will type, given the history of previous typed commands. These predictions (most techniques present a list of the $\mathrm{n}$ most likely commands,

\footnotetext{
${ }^{1}$ although there exist a large number of different Unix shells, the differences between these shells are not relevant for this paper. Therefor we will talk about 'the' Unix command shell
} 
with $\mathrm{n}$ small) are presented to the user, who can accept the prediction or type another command. A simple but useful prediction technique is building a probability table which stores for each command A and B the probability that the next command will be $\mathrm{B}$ given that the last command was A. Many variations on this technique have been investigated: using the $\mathrm{n}$ last commands instead of just the last one or using a decay factor in updating the probabilities to make the most recent information more important than older observations [3]. Other approaches for predicting the next command use decision trees [2] or combine different techniques [9].

These techniques predict up to $75 \%$ of the commands correctly, and about $50 \%$ when also the extra parameters of the command (switches, filenames et cetera) are predicted. The accuracy is even higher when multiple predictions are shown. Although this looks promising, there are some drawbacks:

- the user often uses short commands [6]. For those commands, it is as little effort to type the command as to select or verify a prediction, so nothing is gained.

- long commands are difficult to predict correctly. Verifying and correcting the prediction can take as much time as typing the command (or even more).

- a special shell is necessary to integrate the results of the prediction, but users often want to keep using the shell they are used to.

As a result, these techniques may be very useful to help people who have difficulties with typing (physically impaired or while performing other tasks), but for 'normal' shell users, although it may result in less keystrokes, the time gained will probably be low.

\section{Automating the Automation}

Another way of helping shell users is by suggesting scripts that automate frequent performed tasks. The advantages over the previous approach are:

- the user has to verify the proposed script only once, and can then used it as often as he likes without the need to verify or edit the script.

- even a script that consist of only short commands can still reduce the users effort.

- a system that suggests these scripts can be independent from the shell, so that the user can keep on using his familiar shell.

- it is easy to exchange such proposed scripts with other users.

- users can become more aware of their own behaviour.

This approach also has some drawbacks: probably it does not save you as many keystrokes as the first approach and it takes a large history of typed commands to extract meaningful scripts. Both approaches can be combined, which eliminates some of the drawbacks of the scripting approach, but introduces some drawbacks of the predicting the next command approach. 
To the best of our knowledge, only very few implementations of this scripting approach exist. Graph based induction is used in [11] to analyse which files are used as input or are produced as output by Unix commands. In this way, sequences of commands that operate on one file (or files produced from this file) are detected and transformed into scripts. This allows them e.g. to detect editcompile-run sequences, but they are not able to detect sequences of commands that are not related by common file use such as lpr -P printer $\rangle\langle$ file $\rangle$; $\mathrm{lpq}-\mathrm{P}<$ printer $>$ or sequences of commands that are not related by any resource at all such as starting a set of common applications right after logging in.

Macro operators $[7,8]$ are also related to the approach we presented above. When solving problems one starts from a start state and tries to get to a solution state by applying operators. Macro operators are a sequence of such operators, and the goal is to find a set of such macro operators such that solving the task with the basic operators and the macro operators takes less time than with the basic operators only. This relates to our approach: we look for sequences of actions, and we face the same length consideration: longer sequences solve bigger tasks, but can be used less often. However, there are some major differences between these macro operators and the shell scripts: a script can contain gaps (see section 3.2) and more important the actions in scripts have parameters, which makes this a relational problem.

Why can this be seen as a relational learning problem? Commands are interrelated by their execution order (or time), and each command is possible related to one or more parameters. This allows us to represent a shell $\log$ as a set of logical ground atoms. We translate these as follows:

- commands are translated to stub/3 predicates, where the first argument is the order of execution (which is also a unique identifier), the second argument is the time of execution (expressed as the number of seconds past a certain fixed moment in time) and the third is the command itself.

- parameters are translated to parameter/3 predicates, where the first argument is the identifier of the command, the second argument is the order of the parameter within that command, and the third is the parameter itself.

For example, the shell log

cp /etc/skel/.bashrc /. mybashrc
emacs $\sim$. mybashrc

would be translated to

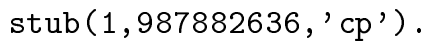

parameter (1,1, '/etc/skel/.bashrc').

parameter (1, 2, ' /.mybashrc').

$\operatorname{stub}(2,987882639$, 'emacs' $)$.

parameter $(2,1$, ' /.mybashrc'). 
Notice that this representation has difficulties representing piped commands. Either such commands must be represented as two separate commands where the first one writes to a imaginary named pipe and the second reads from this named pipe or as one command that has the second command as a parameter. We could also introduce a new predicate to handle this, but this will increase the search space.

We can also represent a parameterised script as a logical conjunction. For instance a script for copying a file and then start editing that copy could be represented as

$\operatorname{stub}\left(A,{ }_{-},{ }^{\prime} c p^{\prime}\right), \operatorname{parameter}(A, 1, F 1), \operatorname{parameter}(A, 2, F 2)$,
$B$ is $A+1, \operatorname{stub}\left(B,,^{\prime}\right.$ emacs'), parameter $(B, 1, F 2)$.

In the next section we present how inductive logic programming can be used to detect such scripts and the problems we faced when applying them to real world data. In section 4 we evaluate the resulting system by analysing shell logs and conclude in section 5 .

\section{Generating Scripts with Warmr}

WARMR [4] is an upgrade of the propositional APRIORI algorithm to an algorithm that can detect association rules expressed in a subset of first order logic. WARMR does this in two phases: first it searches for all frequent patterns (i.e. legal conjunctions of literals as defined in a language) in the dataset, and in a second phase it combines these frequent patterns into association rules. This first phase can more formally be expressed as follows:

Definition 1. Given a set of ground atoms (examples) $\mathcal{E}$, a set of datalog clauses (background knowledge) $\mathcal{B}$, a language $\mathcal{L}$ defining all legal patterns and $m \geq 1$ (minimal occurrence), find all $l \in \mathcal{L}$ such that the query $l$ succeeds for at least $m$ examples in $\mathcal{E} \cup \mathcal{B}$.

The scripts we are looking for can be found with this first phase of the WARMR algorithm. The examples consist of the logs represented as facts as discussed in the previous section, the language allows the stub/3 and parameter $/ 3$ predicates (where these arguments can be constants as well as variables). An additional predicate is added that allows two commands to link to each other. This predicate specifies when two commands are considered next to each other in a sequence. This can be that one command $\mathrm{B}$ must be executed right after command $\mathrm{A}$ (such as in the example above) but it can also be that B is executed at most $\mathrm{n}$ commands after $\mathrm{A}$ or at most $\mathrm{n}$ time units after $\mathrm{A}$. Because we can also provide background knowledge to the system, we can e.g. tell the system that ls -a and ls -all are exactly the same command or tell the system that it should not distinguish between different editor commands. We can also use background knowledge to split up filenames (provided as parameter) into directory, filename root and filename extension. 
In this way we can use WARMR to find the same sequences as those that could be found using the graph induction algorithm, but it has some advantages over the graph induction algorithm:

- additional information can be incorporated via the use of background knowledge (aliases, hierarchies,...),

- any relation between parameters of any command in the sequence can be expressed or learned; not just equality between parameters of two consecutive commands

The major drawback however is the execution time of this algorithm. For a small shell $\log$ of 500 commands, this algorithm needs more than 10 hours $^{2}$ of CPU time to find all sequences that occur at least 5 times. For larger logs the algorithm was even unable to finish due to lack of resources. However we can speedup this algorithm by splitting up the learning task and by using a better algorithm for one of the learning tasks.

\subsection{Speedup by splitting up the learning task}

WARMR builds its frequent patterns levelwise: the list of frequent patterns of level 1 contains all frequent atoms in $\mathcal{L}$. Each run each pattern $P$ in the frequent set is extended to a pattern $P \wedge A$ for each atom $A \in \mathcal{L}$ if there exists no infrequent pattern $Q$ that is more general than $P \wedge A$. This means that WARMr will build patterns that include parameter $/ 3$ atoms for commands in incomplete frequent sequences or commands that will never belong to a frequent sequence. This problem can be solved by first constructing all frequent sequences of commands without taking the parameter information into account and then transform each of these sequences into a separate pattern discovery task to find the frequent parameter patterns. Usually the user is not interested in a script for each frequent pattern, but only in frequent patterns that are not part of a longer frequent pattern, so we filter these out before we start searching for the frequent parameter patterns.

This split up dramatically reduces the size of the search space. Finding the frequent parameter patterns for a given frequent command pattern only takes a few seconds because frequent command patterns only contain few commands (compared with the total set of commands) and so the search space is small. Most of the time still goes into finding frequent command patterns. But since this is a propositional task, more efficient algorithms can be found.

\subsection{Speedup by using the minimal occurrence algorithm}

The way in which WARMR extends a frequent pattern is not optimised for finding frequent sequences in which the order of the items is important. We illustrate this with an example. Suppose that our dataset contains the frequent sequence

\footnotetext{
${ }^{2}$ all timings in this paper are on a Pentium III $800 \mathrm{MHz}$ computer with $256 \mathrm{Mb}$ memory, running linux and the Ace version of WARMR running ilProlog[1]
} 
a b c d. When WARMR has reached its second level, it has constructed these frequent patterns:
$\mathrm{a} \rightarrow \mathrm{b}$
$b \rightarrow c$
c $\rightarrow d$

However, when WARMR extends the first pattern $a->b$, it will extend it to
$\mathrm{a} \rightarrow \mathrm{b} \rightarrow \mathrm{a}$
$\mathrm{a} \rightarrow \mathrm{b} \rightarrow \mathrm{b}$
$\mathrm{a} \rightarrow \mathrm{b} \rightarrow \mathrm{c}$
$\mathrm{a} \rightarrow \mathrm{b} \rightarrow \mathrm{d}$

and test each of these patterns on the dataset, to see that only the third succeeds. However, since we are looking for subsequent commands, we know that only a -> $\mathrm{b} \rightarrow \mathrm{c}$ can be a valid extension. Moreover, if we know the identifiers at which the sequences $a \rightarrow b$ and $b \rightarrow c$ start, we can calculate from this the number of occurrences of $a \rightarrow b \rightarrow c$ (together with the identifiers of a on which these sequences start) without looking into the dataset.

The above idea is implemented in the minimal occurrence algorithm [10]. We use an algorithm based on this minimal occurrence algorithm to find all maximal frequent sequences, where we add identifiers for left and right parents to efficiently combine elements into longer sequences.

Definition 2. Let $s$ be a sequence of $n$ elements: $e_{s, 1} e_{s, 2} e_{s, 3} \ldots e_{s, n-1} e_{s, n}$. A sequence $s^{\prime}\left(e_{s^{\prime}, 1} e_{s^{\prime}, 2} \ldots e_{s^{\prime}, p-1} e_{s^{\prime}, p}\right)$ of length $p$ is a subsequence of the sequence $s$ of length $n$ if

$1 \leq p \leq n$

and $\forall i 1 \leq i \leq p, \exists j 1 \leq j \leq n: e_{s^{\prime}, i}=e_{s, j}$

and $\forall i, j 1 \leq i<j \leq \bar{p} \exists k, l 1 \leq k<l \leq n: e_{s^{\prime}, i}=e_{s, k}$ and $e_{s^{\prime}, j}=e_{s, l}$.

The frequency of a subsequence in a sequence is the number of different mappings from elements of $s^{\prime}$ into the elements of s such that the previous conditions hold.

Notice that this a general definition, usually we will restrict the sequence by introducing a maximal gap size in the third requirement:

$\forall i 1 \leq i<p \exists k, l 1 \leq k<l \leq n, l-k \leq$ gapsize $+1: e_{s^{\prime}, i}=e_{s, k}$ and $e_{s^{\prime}, i+1}=e_{s, l}$

Definition 3. A sequence $s^{\prime}$ is a maximal subsequence of frequency $f$ of a sequence $s$ if $s^{\prime}$ is a subsequence of the sequence $s$ with a frequency of at least $f$ and there is no sequence $s^{\prime \prime}$, subsequence of $s$ with frequency at least $f$, such that $s^{\prime}$ is a subsequence of $s^{\prime \prime}$.

Each sequence $s$ of length $n>1$ has 2 frequent subsequences of length $n-1$. We call this first subsequence le ftparent $(s)$ and the second rightparent $(s)$. To extend a sequence $s$ of length 2 or longer, one just combines this sequence with a sequence $t$ where the right parent of $s$ is the left parent of $t$. However for 
sequences $p$ and $q$ of length one, there are no restrictions in combining them, except that $p$ should occur before $q$ (and the maximal gap size if defined). Because of this, for constructing the second level, we can do no better than just trying all combinations of 2 sequences from level one. The algorithm is shown in figure 1 . We give each sequence a unique identifier and we store and index the identifiers from left and right parents for each sequence. This allows us to retrieve possible extensions for a frequent subsequence very efficient.

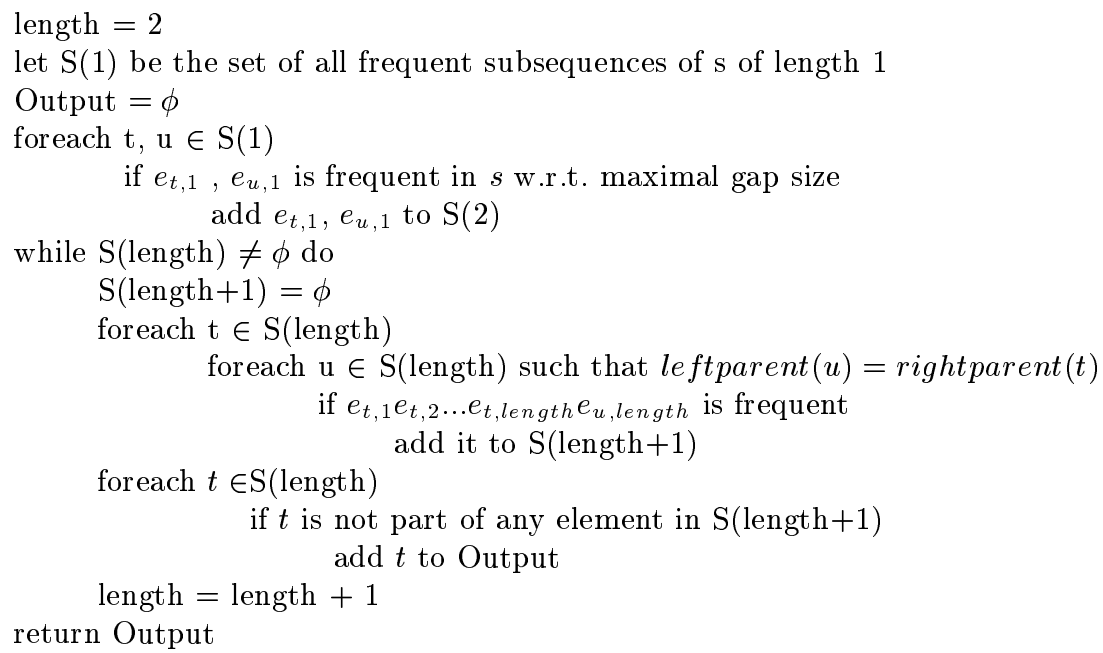

Fig. 1. Calculating all frequent maximal subsequences of $s$

This algorithm is also related to the cSPADES algorithm [12] which can search for sequences in large databases in an efficient way. However, we combine elements based on their left and right parent instead of combining elements with identical tails because this last approach results in a less efficient search when using maximal gap size constraints as we do.

\subsection{Gluing everything together}

How do we use all this information to transform a shell log into a set of shell scripts? First we remove from the logs all commands that are useless in scripts because they can not change the state of the computer: 1s, more, date et cetera. Of course, when the aim of the analysis is to get an insight in the behaviour of the user instead of producing scripts, this step can be skipped.

Next we use our variant of the minimal occurrences algorithm on the altered $\log$ file. By varying the maximal gap size and the minimal frequency for a subsequence to be considered frequent one can control the number of frequent sequences. We also add to the algorithm the possibility do define constraints on 
the subsequences. These constraints allow us for example to reject sequences that use a command more than $n$ times. We output the frequent subsequences as a set of freqep/4 predicates, where the first argument is the identifier of the subsequence, the second is the identifier of the mapping (the highest identifier for a given subsequence is the frequency of that subsequence), the third argument is the element identifier within the subsequence and the fourth argument is the element identifier within the input sequence. We use this representation because it is independent of the ILP program that is going to use this subsequence information.

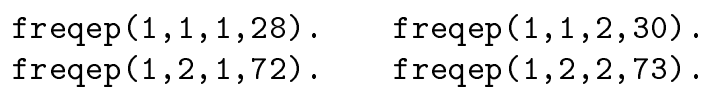

This could be such a result from this algorithm, specifying that it found only one frequent subsequence, that occurred twice: element number 28 in the input sequence is the first element in the first occurrence of the subsequence, element 30 is the second (so there is a gap of size 1). The second occurrence of the sequence is at position 72 and 73 .

In the next step we add the subsequence output to the log data that we transform to the representation discussed in section 2 . We do not allow stub/3 atoms to be used in the language (WARMR would again start looking for frequent sequences this way) but force the use of freqep/4 instead. The parameter $/ 3$ and other predicates defined in background knowledge are also added to the language. We then let WARMR search for frequent patterns. An example of such a pattern is:

freqep (1,0cc,1,Pos1), parameter(Pos1,1,Param1), base(Param1,Base1), ext (Param1, 'tex'), freqep(1,0cc, 2,Pos2), parameter(Pos2,1, Param2), ext (Param2,'dvi'), base(Param2, Base1).

Since WARMR outputs all frequent patterns, we first have to make sure we remove all redundant rules. For instance together with the above rule, WARMRwould also produce next rules:

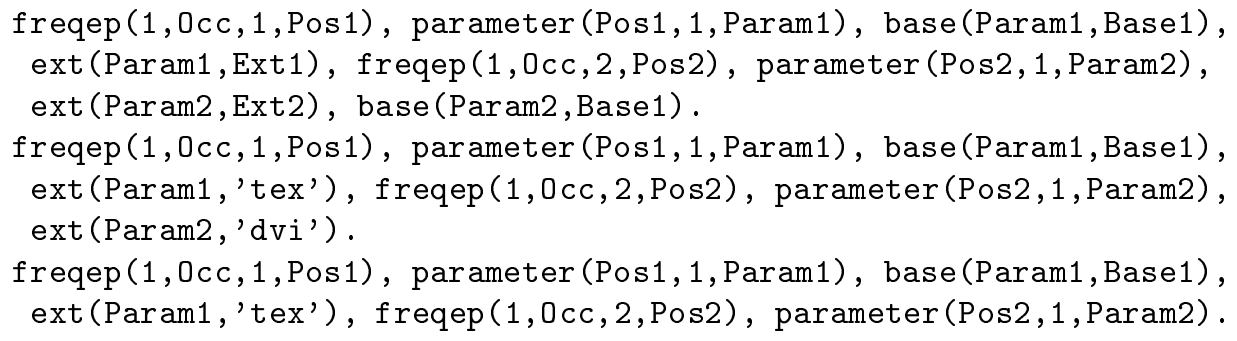

et cetera.

Next the most specific frequent rules are translated to scripts: the commands are looked up in the input sequence and the remaining of the rules is parsed and written as a Unix function. Our first rule would be translated to: 


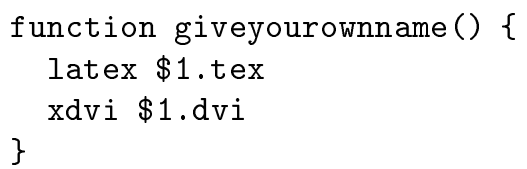

Now we sort the resulting scripts. Although length and frequency are important attributes of a script, we sort the scripts according to the frequency divided by the expected frequency of this subsequence in a sequence where the elements are put in random order. The higher this number, the more 'exceptional' the sequence is.

\section{Experiments}

We compare these approaches on the Greenberg dataset [5]. Table 1 shows the timings of finding frequent subsequences for 5 user logs from the Greenberg dataset with the default minimal frequency of 0.01 . We see that, as the number of commands grows, the execution time of both algorithms (expressed in seconds) increases. The number of maximal frequent subsequences found does not seem to influence the execution time. The minimal occurrences algorithm is about 7 times faster than WARMR.

\begin{tabular}{|c|c|c|c|c|c|}
\hline file & \#commands & \#frequent seq. & max. length seq. & time Warmr & time minocc \\
\hline non-5 & 216 & 4 & 6 & 1.2 & 0.5 \\
non-16 & 590 & 26 & 6 & 13.8 & 1.8 \\
non-23 & 962 & 21 & 3 & 21.7 & 2.0 \\
non-11 & 1537 & 13 & 4 & 30.4 & 4.5 \\
non-4 & 3608 & 16 & 4 & 83.7 & 13.1 \\
\hline
\end{tabular}

Table 1. Timing results with maximal gap size 0

However, when we set the maximal gap size to 1 , this changes the timings dramatically (see table 2). It takes WARMR almost 200 times as long as with gap size 0 to find the sequences for non-5. For non-16 this is nearly a factor 800 slower. For larger datasets, WARMR was not able to compute all sequences due to lack of resources. The minimal occurrences algorithm does not suffer from this problem, it does not even double the execution time.

When we raise the maximal gap size to 2 (see table 3 ) WARMR is not able to find all frequent subsequences for even the smallest log file. While the other algorithm finishes in about a minute or less, we see that not only the length of the input but also the number of frequent subsequences found influences the execution time.

Just for illustration purpose we show some of the scripts found:

$\begin{array}{llll}1 \mathrm{p} r & -\mathrm{P} & \$ 1 & \$ 2\end{array}$

cd . .

rmdir $\$ 1$ $\operatorname{mkdir} \$ 1$

cd $\$ 1$ 


\begin{tabular}{|c|c|c|c|c|}
\hline file & \#frequent seq. & max. length seq. & time Warmr & time minocc \\
\hline non-5 & 8 & 6 & 236 & 0.7 \\
non-16 & 70 & 9 & 9975 & 2.6 \\
non-23 & 31 & 6 & - & 2.4 \\
non-11 & 63 & 8 & - & 6.9 \\
non-4 & 39 & 6 & - & 18.2 \\
\hline
\end{tabular}

Table 2. Timing results with maximal gap size 1

\begin{tabular}{|c|c|c|c|}
\hline file & \#frequent seq. & max. length seq. & time minocc \\
\hline non-5 & 35 & 9 & 1.6 \\
non-16 & 607 & 13 & 27.1 \\
non-23 & 99 & 9 & 5.6 \\
non-11 & 401 & 11 & 40.6 \\
non-4 & 173 & 10 & 63.6 \\
\hline
\end{tabular}

Table 3. Timing results with maximal gap size 2

\section{Conclusion}

In this paper we tackled the problem of creating shell scripts (a sequence of commands together with their (variable) parameters) from shell logs. Since this task can be formulated as a relational pattern discovery task, we used the WARMR algorithm. To speed up this task we separate the propositional subtask (detecting frequent subsequences of command stubs) from the relational task (detecting frequent patterns in the parameters of a frequent subsequence of commands). We also presented a version of the minimal occurrences algorithm to find frequent subsequences in an efficient way.

We compared WARMR with this new system on real world data. WARMR is considerably slower, and often fails to find all sequences due to lack of computing resources. These experiments also show that shell logs do contain frequent subsequences, and even frequent subsequences of considerably length.

Other possible applications for this technique are other sequence analysing tasks, such as analysing traces of visits to a website where each click is annotated with extra information. This can help the designer in understanding how users visit the site or allow for automatic site modification. The system can also be used in analysing other user interfaces: a prototype of the user interface is built and testers try this interface. All their annotated actions are logged and analysed. If important long subsequences of actions are found, the developer can consider redesigning the interface in such a way that these actions can be performed more easily. All applications were sequential annotated data is involved and were frequent subsequences provide important knowledge are potential application domains for this technique.

But not only in applications where detecting sequences is the main task, also other applications can make use of efficient subsequence detection algorithms. 
Analysis of molecular data is such an example: sequences of atoms can be important features in such applications.

Acknowledgements: The authors thank Jan Ramon for his help with the WarmR system, Luc De Raedt for the discussions on this subject and Saul Greenberg for providing the test data. Hendrik Blockeel is a post-doctoral fellow of the Fund for Scientific Research of Flanders (FWO-Vlaanderen).

\section{References}

1. H. Blockeel, B. Demoen, L. Dehaspe, G. Janssens, J. Ramon, and H. Vandecasteele. Executing query packs in ILP. In J. Cussens and A. Frisch, editors, Proceedings of the 10th International Conference in Inductive Logic Programming, volume 1866 of Lecture Notes in Artificial Intelligence, pages 60-77, London, UK, July 2000. Springer.

2. Brian D. Davison and Haym Hirsch. Experiments in UNIX command prediction. In Proceedings of the 14 th National Conference on Artificial Intelligence and 9th Innovative Applications of Artificial Intelligence Conference (AAAI-97/IAAI-97), pages 827-827, Menlo Park, July 27-31 1997. AAAI Press.

3. Brian D. Davison and Haym Hirsh. Predicting sequences of user actions. In Predicting the Future: AI Approaches to Time Series Problems, pages 5-12. AAAI Press, July 1998. WS-98-07.

4. L. Dehaspe. Frequent Pattern Discovery in First-Order Logic. PhD thesis, Department of Computer Science, Katholieke Universiteit Leuven, 1998. http: //www.cs.kuleuven.ac.be/ $1 \mathrm{dh} /$.

5. Saul Greenberg. Using unix: collected traces of 168 users. Technical report, University of Calgary, 1988.

6. Saul Greenberg. The Computer User as Toolsmith: The Use, Reuse, and Organization of Computer-Based Tools. Cambridge Series on Human-Computer Interaction. Cambridge University Press, 1993. QA76.9H85G73.

7. Glenn A. Iba. A heuristic approach to the discovery of macro-operators. Machine Learning, 3:285-317, 1989.

8. R. E. Korf. Learning to Solve Problems by Searching for Macro-Operators. Research Notes in Artificial Intelligence. Pitman, London, 1985.

9. Benjamin Korvemaker and Russell Greiner. Predicting UNIX command lines: Adjusting to user patterns. In Adaptive User Interfaces: Papers from the 2000 AAAI Spring Symposium, pages 59-64, 2000.

10. Heikki Mannila and Hannu Toivonen. Discovering generalized episodes using minimal occurrences. In Evangelos Simoudis, Jia Wei Han, and Usama Fayyad, editors, Proceedings of the Second International Conference on Knowledge Discovery and Data Mining (KDD-96), page 146. AAAI Press, 1996.

11. Hiroshi Motoda and Kenichi Yoshida. Machine learning techniques to make computers easier to use. Artificial Intelligence, 103(1-2):295-321, 1998.

12. Mohammed Javeed Zaki. Sequence mining in categorical domains: Incorporating constraints. In Arvin Agah, Jamie Callan, and Elke Rundensteiner, editors, Proceedings of the 2000 ACM CIKM International Conference on Information and Knowledge Management (CIKM-00), pages 422-429, N.Y., November 6-11 2000. ACM Press. 